\title{
Engineering Geological Evaluation Study of a Proposed Solid Waste Landfill Site
}

\author{
BahaAeldin H. Sadagah \\ Department of Engineering \& Environmental Geology Faculty of \\ Earth Sciences, King Abdulaziz University, Jeddah Saudi Arabia \\ P.O. Box 80206, Jeddah 21589, Email: bsadagah@kau.edu.sa
}

Received: 21/12/2003 Revised: 21/3/2004 Accepted: 3/5/2004

\begin{abstract}
Intensive site location operation was conducted in the western region, to locate a site suitable for the disposal of solid wastes to serve an urban district of about 800,000 inhabitants. This study investigates the engineering geological properties of the proposed landfill site, Malkan-1. The site is formed of Precambrian basement rocks, covered by alluvial deposits and sand dunes, surrounded by a low to medium relief topography. The site is of about $1.2 \mathrm{~km}$ long and $800 \mathrm{~m}$ wide includes single outlet to the main wadi. Geological, structural features and engineering geological parameters were studied, in addition to intensive investigation of the prevailing joints system. These collected data were implement to the rock mass classification systems, and conclude the suitability of the site to be used as a solid waste landfill.
\end{abstract}

\section{Introduction}

In the last decade, considerable attention has been paid to the environmental problems and/or pollution and the assessment of their impact on human, especially in the Kingdom of Saudi Arabia. As a result of increased environmental awareness, questions regarding the geological barriers and geoenvironmental effects of waste disposal and waste contamination are a general issue. More emphasis has been placed on multidisciplinary site investigations and landfill site evaluation. For this reason, a new guide for a site selection, investigation and evaluation for waste purpose has been published by Langer 
(1995). Morfeldt and Langer (1989) gave information on the possibilities and restrictions of underground waste repositories.

The suitability of the geological medium for final disposal can only be demonstrated if a comprehensive safety analysis has shown that the interaction of the system "waste product/final disposal facility/overall geological situation" can maintain the predetermined protection aims. The product to be disposed of and the engineering concept of the deposit facility on one hand, and the condition of the geological formations on the other hand, mutually interact and simultaneously place requirements on each other.

The challenge is to ensure the proper disposal of substances that must be prevented from returning to the biosphere in even minute quantities, during time periods of almost geological timescale. This means that national considerations of general guidelines should exist at the international level according to an internationally accepted "code of practice".

The interaction between engineering geology, soil mechanics and rock mechanics has attained today such a level as to ensure efficiency. From the engineering geological point of view, any disposal site for highly toxic wastes must be chosen so that the transport of dangerous quantities of toxic particles into the biosphere via circulating groundwater can be avoided. In principle, all final disposal concepts should be safeguarded by a system of parallel or interlocking natural and technical barriers (multi-barrier principle), although the effectiveness of such technical and natural barriers may receive different weighting in different disposal concepts.

\section{Location of the Study Area}

Wadi Malkan lies at a suburban area (Fig. 1). The location is a preliminary study of a proposed landfill site to serve a $20 \mathrm{~km}$ away urban area of almost 800,000 inhabitants producing about 2000 tons/day of solid wastes. Malkan disposal site lies at the west bank of Malkan valley, named as Malkan-1 (Fig. 1). The site formed of a narrow valley intruded the plutonic rock masses for about one kilometer, and branches in the site into a number of small wadis of about $20 \mathrm{~m}$ widths. All the small tributaries in the site have a single main outlet at the east side to Wadi Qashaa. The site branched from the west side of Wadi Qashaa, which in turn branch from the west side of the main valley in the area, Wadi Malkan. This geomorphologic situation adds a privilege to the site to be chosen as a solid waste disposal site. Contour lines of the location have been digitized using computer program Didger and fed to the computer program Surfer to generate a three-dimensions model of the site (Fig. 2). 


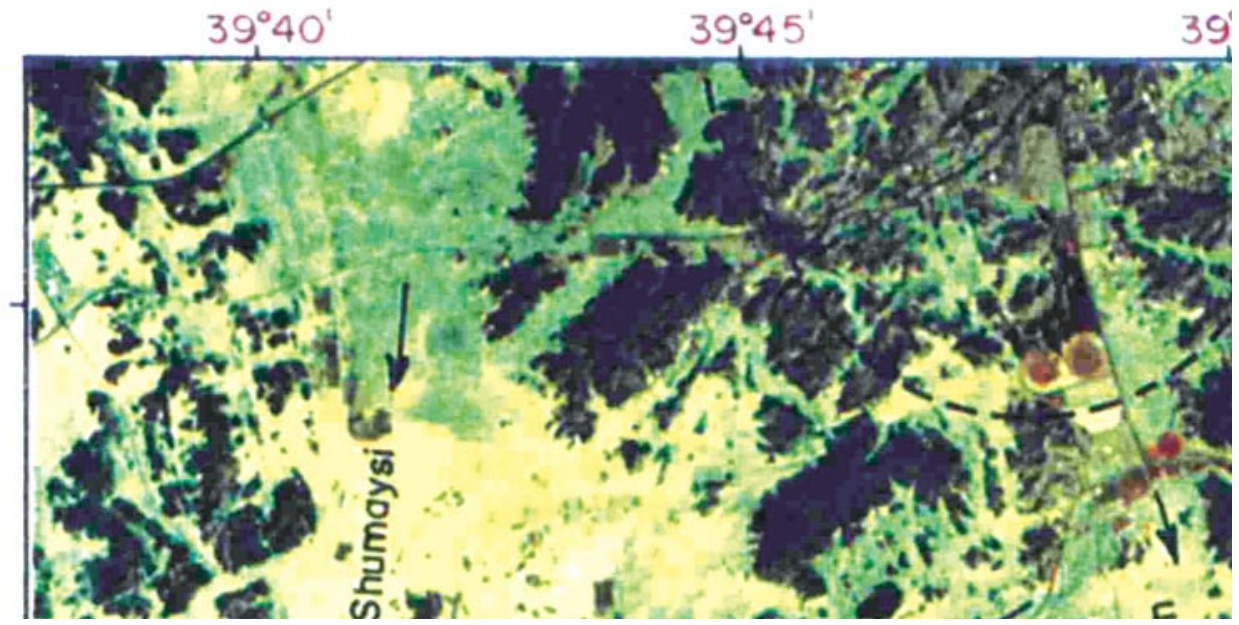

FIG. 1. Location map of Malkan waste disposal landfill site (after Mokhtar et al., 1995).

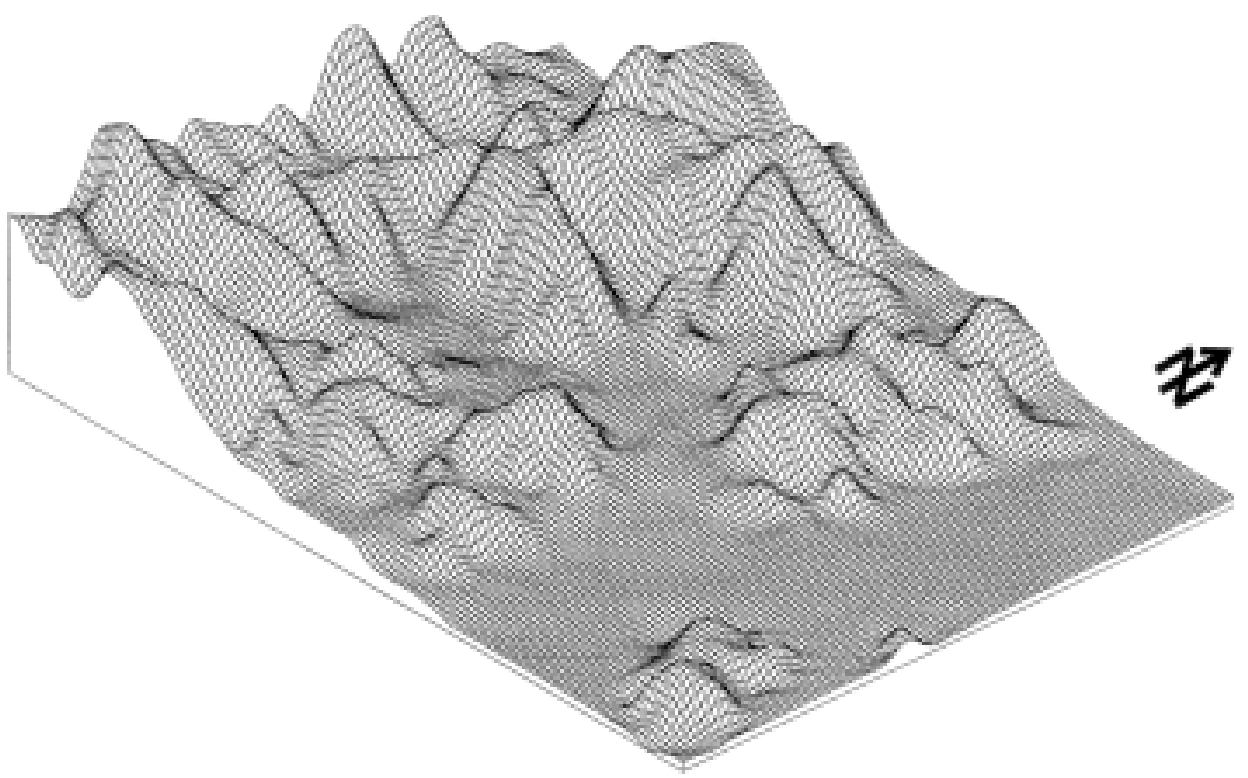

FIG. 2. Surface plot map of Malkan-1 proposed waste disposal site. 


\section{Geology}

The rock exposures in the investigated site formed of igneous and metamorphic rocks. Thin section study indicates the names of the rocks as quartz diorite (tonalites), hornblendite, gabbro, hornblende gabbro (norite), and biotite tonalite (Fig. 3). These rocks are mainly dark-color medium- to coarse-grained intrusives, intruded the green schist country rocks (Moore and Al-Rehaili, 1989), which could be of sedimentary origin. Along the geologic history, the country rocks are eroded, and the intrusions are now forming the relief surface. Relics of the country rocks (quartz diorite) are located north of the study site. Rock masses are forming hills of low to medium elevation toward the center of the site to fairly high elevation hills surrounding the site, towards north and west (Fig. 2). Dikes of andesite, mylonite, and aplite located randomly in the area strike northeast-southwest, showing a definite structural pattern, younger in age than the hosted rock masses intrusives (diorites and gabbros), and parallel to the prevailing joint sets.

\section{Structural Pattern}

\section{Joint sets}

The joints sets in the area were studied carefully. The area was divided into 18 stations. In each station, the attitude of hundred joints planes were measured in order to recognize the attitude of the prevailing joint sets in each station, using Dips computer program (Fig. 4). The trend of the joint sets shows a definite trend of northeast and north-northwest joint sets. Where schistosity is towards northeast, roughly parallel to the dikes attitude.

\section{Faults}

A few numbers of faults are located in the area. These faults are of smallscale size. An example of strike-slip faults is shown in stations 4 and 8 . These faults could be related to the tectonic forces that built the Arabian Shield at the Oligocence age. Site investigation shows that there are no regional faults observed in the proposed waste disposal site, where the landfill site is full of shallow alluvial deposits (Mokhtar et al., 1995).

\section{Shear zones}

The shear zones are located following no definite structural pattern. For example, i) station 4 shows two shear zones, the first of $8 \mathrm{~m}$ long and $50 \mathrm{~cm}$ wide of attitude 20/20, and the second of $10 \mathrm{~m}$ long and $50 \mathrm{~cm}$ wide of attitude 50/92, ii) station 16 shows a shear zone of $60 \mathrm{~cm}$ width and $10 \mathrm{~m}$ long, iii) station 5 shows a shear zone of width $50 \mathrm{~cm}$ and $10 \mathrm{~m}$ long of attitude $50 / 180$, and iv) 


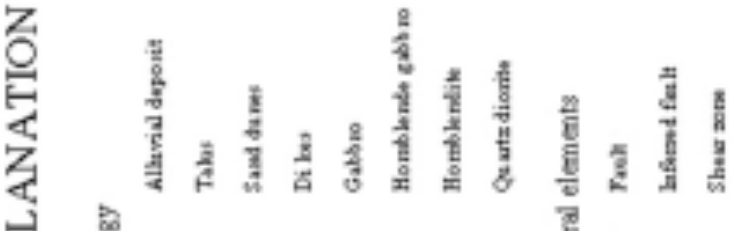

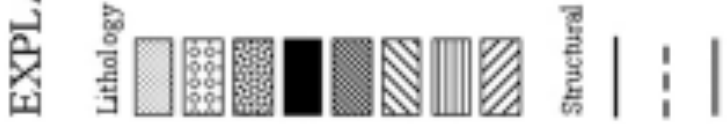

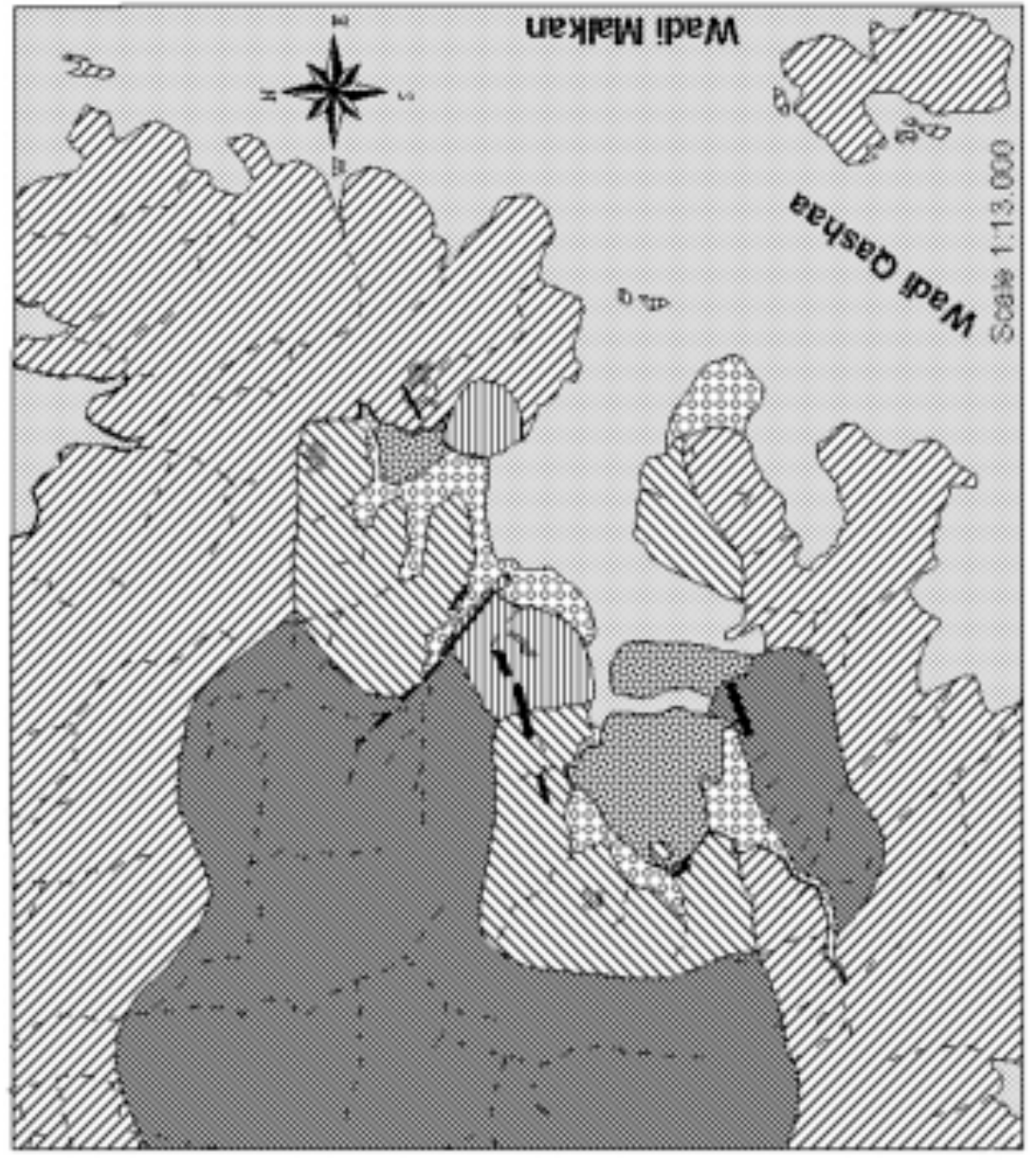

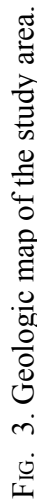




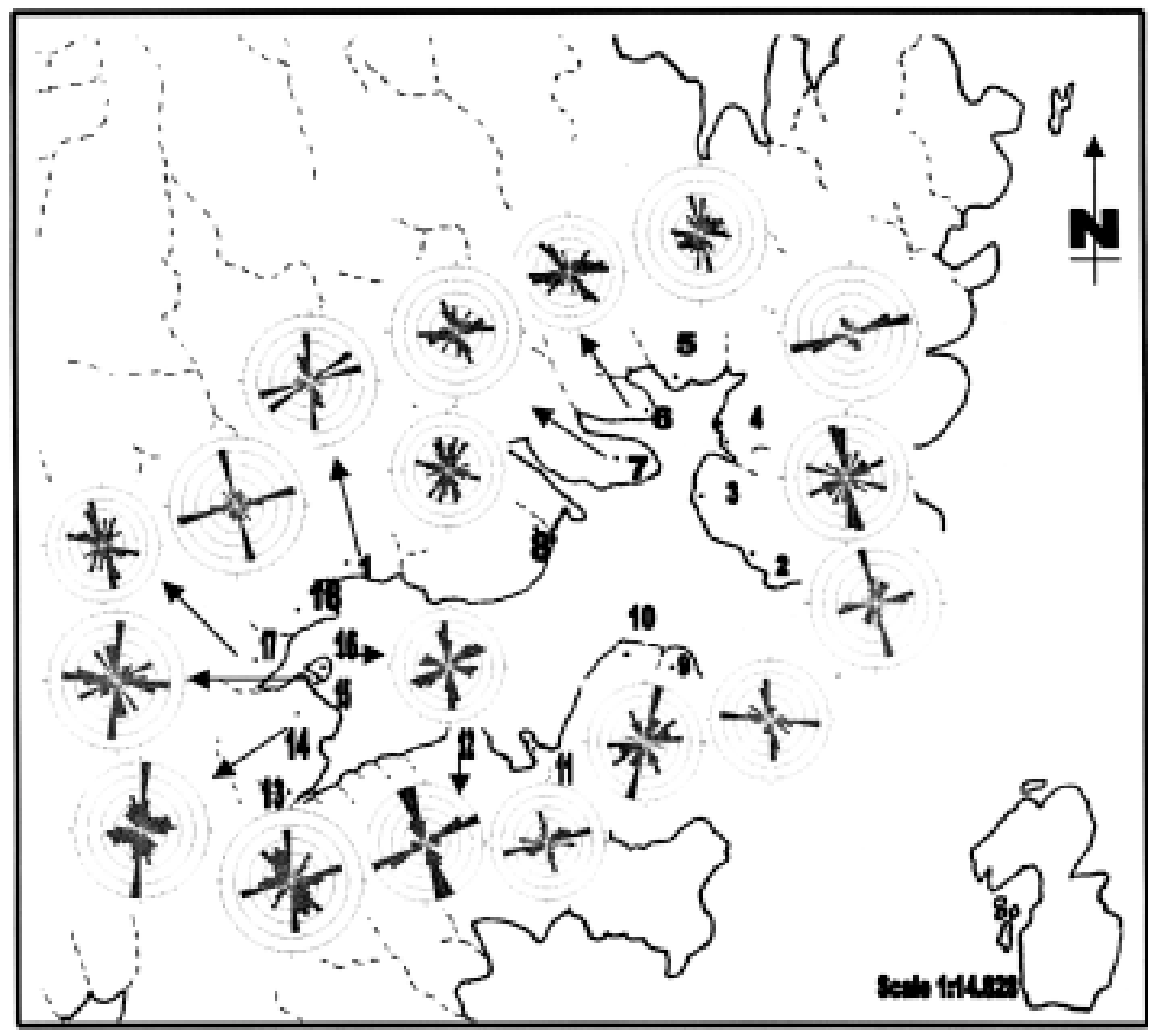

FIG. 4. Rose diagrams of joints orientation at 18 stations in the study area.

station 8 shows a shear zone of $10 \mathrm{~m}$ long and $60 \mathrm{~cm}$ wide of attitude 35/125. These shear zones are of different attitudes, parallel to the prevailing joint sets at each station (Fig. 4).

\section{Dikes}

A few dikes of mylonite and andesite are scattered in the area, following the attitude of the joint sets. The dikes length is about $30 \mathrm{~m}$ long and $1.5 \mathrm{~m}$ thicknesses.

\section{Site Investigation}

A preliminary site investigation of the proposed landfill (Malkan-1) was carried out in order to determine the site geology, the position of the water table, the permeability, in addition to the relevant engineering properties of both rock 
masses and soils concerned. The position of the water table and the permeability of the rocks and soils are particularly important in relation to the possible pollution of groundwater by leachate produced by the disintegration of the solid waste.

In a parallel study, geophysical investigation had been carried out in the site to indicate the depth to detect i) the groundwater level and ii) the presence of faults under the soil cover (Mokhtar, et al., 1995 and 1996).

The site was divided into 18 stations, where possible change in the engineering properties of the rock masses is expected. In each of these stations, technical investigation and characterization of the rock masses and rock materials as well as soils were carried on.

\section{Engineering Geological Characteristics of the Site}

\section{Rock material and rock mass properties}

Due to limited properties are required, so some of the technical properties of the rocks were only studied. These index properties are given below:

\section{Density}

A number of seven lump samples were collected from each station over the studied site. The density of each lump was measured in the laboratory using Archimedes method (ASTM, 1995) and the average density for each rock type is recorded (Table 1).

\section{Point load}

A number of seven lump samples were collected from each station over the studied site. The uniaxial strength, $\sigma_{c}$, of each lump was indirectly calculated by the equation measured in the laboratory using the point load apparatus. The average strength index, $I_{s}$, of the number of samples related to the same rock type is recorded. Using the following equation given by Broch and Franklin (1972):

$$
I_{s}=\frac{P}{D^{2}}
$$

where $\mathrm{P}$ is the load required to break the specimen and

$\mathrm{D}$ is the diameter of the core at failure

The indirect relationship between the uniaxial compressive strength, $\sigma_{c}$, and the point load index, $I_{s}$, (Table 1) is given by the following equation given by Bieniawski (1975): 


\begin{tabular}{|c|c|c|c|c|}
\hline 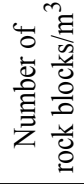 & तิ $\quad \frac{n}{6}$ & 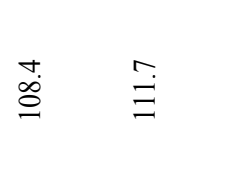 & $\stackrel{\Upsilon}{=} \quad \stackrel{2}{=}$ & $\stackrel{+}{=} \quad \stackrel{\infty}{=}$ \\
\hline $\begin{array}{l}\dot{\vec{z}} \\
\dot{\vec{\nu}}\end{array}$ & 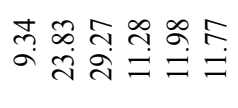 & 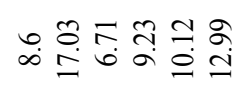 & 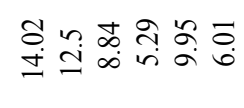 & 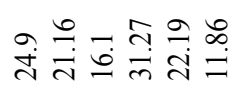 \\
\hline $\begin{array}{l}\text { E } \\
\text { : } \\
\text { : } \\
\stackrel{0}{0}\end{array}$ & 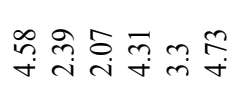 & 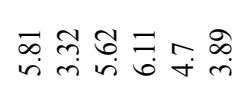 & 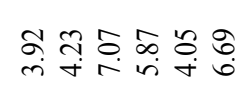 & 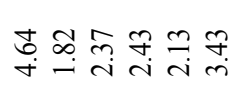 \\
\hline$\stackrel{\infty}{\gtrless^{\infty}} \approx$ & $\begin{array}{ll}\infty & \approx \\
\sigma & \infty\end{array}$ & 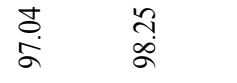 & 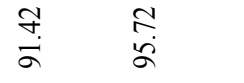 & $\begin{array}{ll}\bar{\infty} & 0 \\
\circ & \sigma\end{array}$ \\
\hline 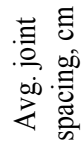 & 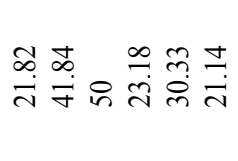 & 궁 & 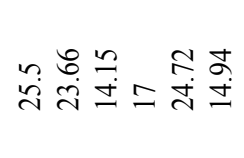 & 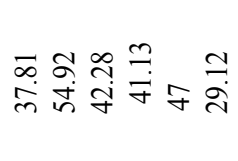 \\
\hline$\approx$ & 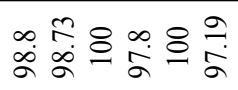 & 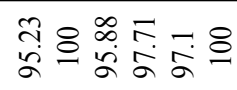 & 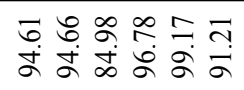 & 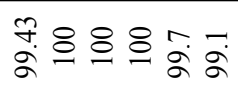 \\
\hline 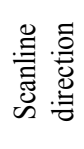 & 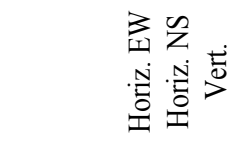 & 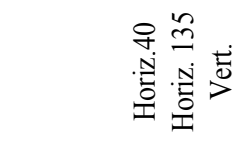 & 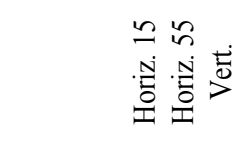 & 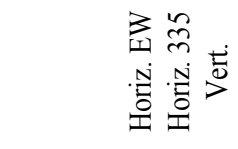 \\
\hline 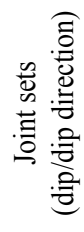 & 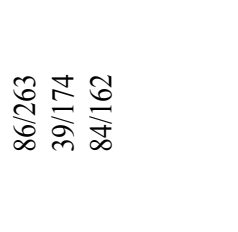 & 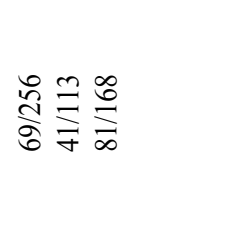 & 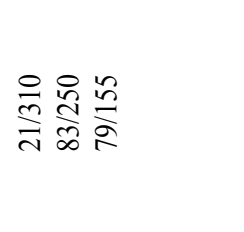 & 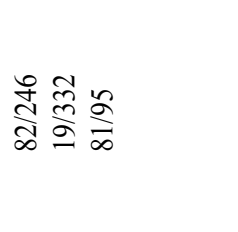 \\
\hline $0^{0} \sum^{\pi}$ & ণ̌ & 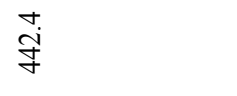 & ்ָֻ & $\hat{\Omega}$ \\
\hline$\beta^{n}$ & $=$ & 音 & $\stackrel{\Xi}{\Xi}$ & 声 \\
\hline 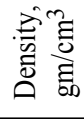 & $\stackrel{i}{i}$ & ते & ते & $\stackrel{\Re}{i}$ \\
\hline 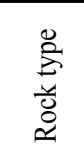 & $\begin{array}{l}\text { 유 } \\
\text { 芯 } \\
\dot{0} \\
\dot{1}\end{array}$ & 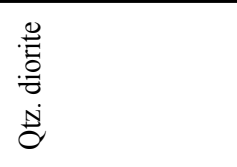 & 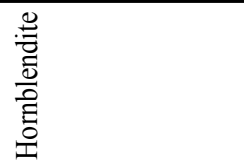 & 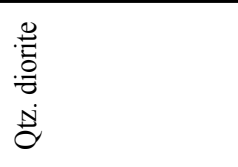 \\
\hline 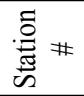 & - & $\sim$ & $m$ & $\nabla$ \\
\hline
\end{tabular}




\begin{tabular}{|c|c|c|c|c|}
\hline 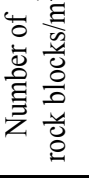 & $\ddot{\vec{q}}$ & $\begin{array}{ll}\infty \\
\infty \\
\infty\end{array} \quad \stackrel{J}{\sigma}$ & $\stackrel{0}{\vec{m}}$ & 崩 \\
\hline $\begin{array}{l}\dot{\vec{d}} \\
\dot{\vec{D}}\end{array}$ & 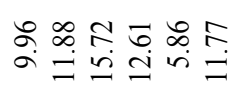 & 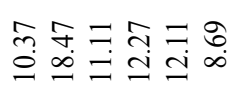 & 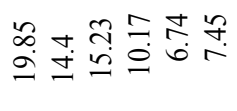 & 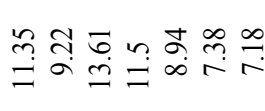 \\
\hline 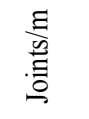 & 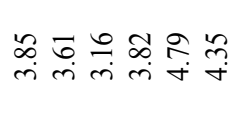 & 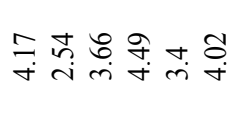 & 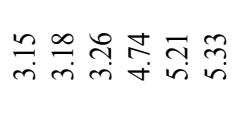 & 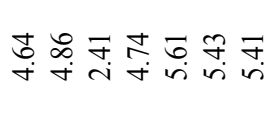 \\
\hline$\stackrel{\text { in }}{\gtrless} \approx$ & $\begin{array}{ll}n & \overline{0} \\
2 & \infty\end{array}$ & \begin{tabular}{ll}
0 & $\infty$ \\
\hdashline & $n$ \\
$a$ & $a$
\end{tabular} & $\begin{array}{ll}\sqrt{n} & \infty \\
a & \stackrel{5}{a}\end{array}$ & 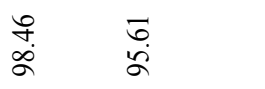 \\
\hline 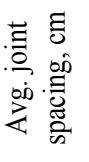 & 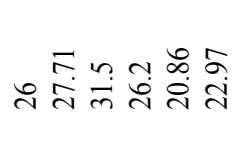 & 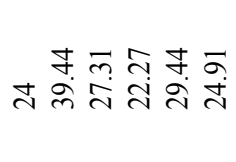 & 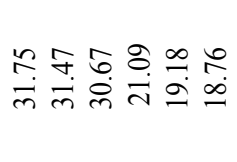 & 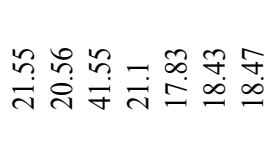 \\
\hline$\approx$ & \&ڤ \& & - \& & 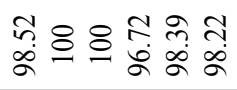 & 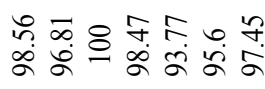 \\
\hline 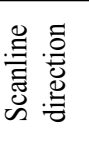 & 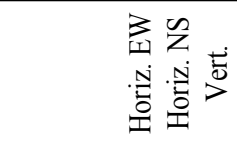 & 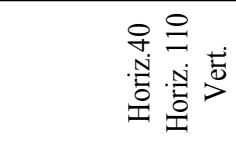 & 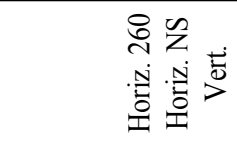 & 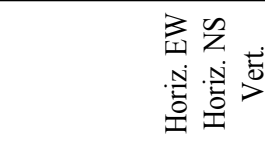 \\
\hline 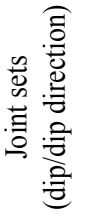 & 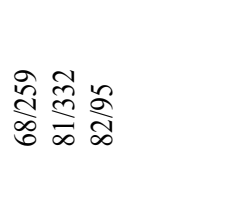 & 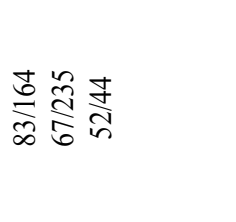 & 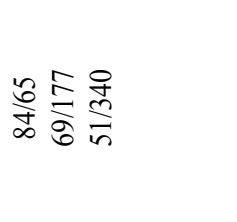 & 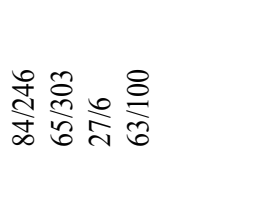 \\
\hline $0^{0} \tilde{\Sigma}$ & 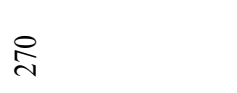 & $\underset{\infty}{\stackrel{\infty}{\Xi}}$ & $\begin{array}{l}\infty \\
\infty \\
\infty \\
\infty\end{array}$ & $\stackrel{\underset{+}{\sim}}{\sim}$ \\
\hline 3 & 罟 & 䍐 & 䍐 & $\equiv$ \\
\hline 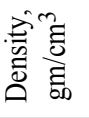 & a & $\hat{\text { }}$ & $\underset{\text { ç }}{\tilde{r}}$ & ふิ \\
\hline $\begin{array}{l}\stackrel{0}{0} \\
\stackrel{0}{0} \\
\stackrel{0}{0}\end{array}$ & 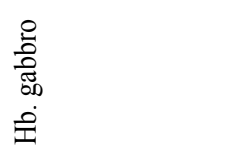 & 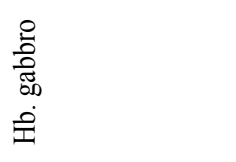 & 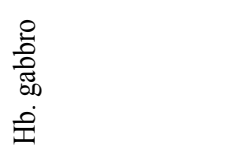 & 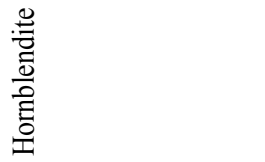 \\
\hline 惹 & in & 0 & $r$ & $\infty$ \\
\hline
\end{tabular}




\begin{tabular}{|c|c|c|c|c|}
\hline 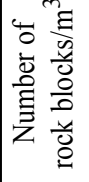 & ڤె. & 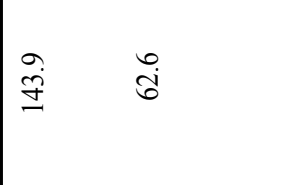 & 卞 & $\underset{\infty}{\tilde{D}}$ \\
\hline 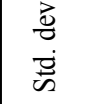 & 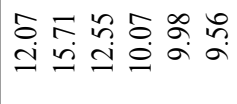 & 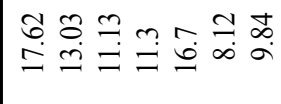 & 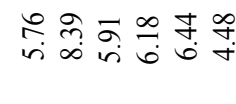 & 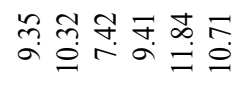 \\
\hline 롤 & 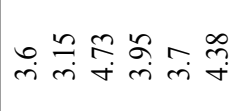 & 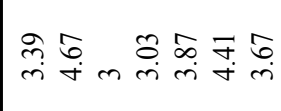 & 者 金 & 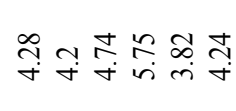 \\
\hline 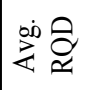 & $\begin{array}{ll}\vec{\infty} & \frac{J}{+} \\
\dot{\alpha} & \infty\end{array}$ & 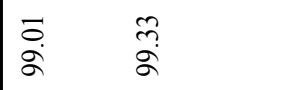 & $\begin{array}{ll}\infty & \stackrel{\sigma}{\tau} \\
\stackrel{a}{a}\end{array}$ & $\begin{array}{ll} \pm & i \\
a & a\end{array}$ \\
\hline 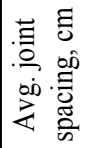 & 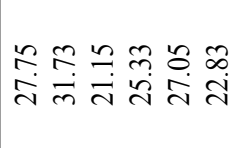 & 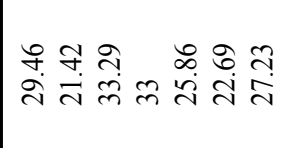 & 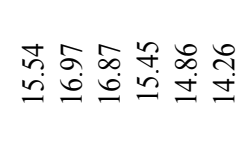 & 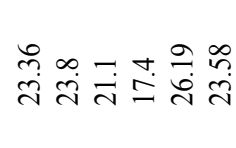 \\
\hline$\approx$ & 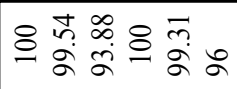 & $\begin{array}{l}\tilde{a} \\
\alpha \\
\alpha\end{array}$ & 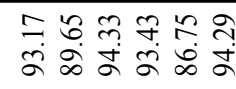 & 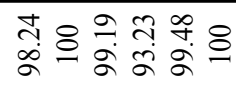 \\
\hline 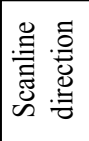 & 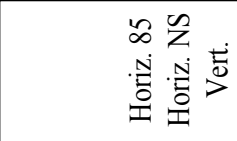 & 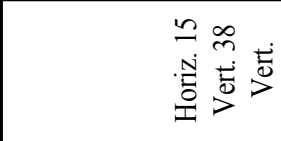 & 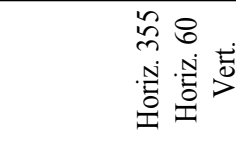 & 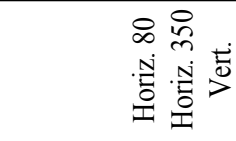 \\
\hline 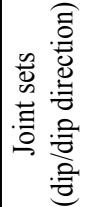 & 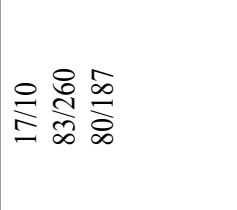 & 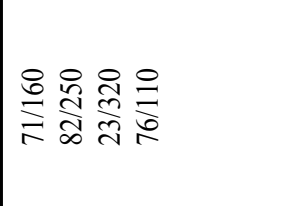 & 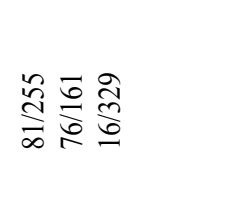 & 壳 \\
\hline $0^{0} \stackrel{\tilde{\Sigma}}{\Sigma}$ & 吕 & 年 & $\dddot{2}$ & $\stackrel{\infty}{\stackrel{\infty}{\sim}}$ \\
\hline $3^{n}$ & 夆 & 音 & $\sum_{\Xi}$ & 声 \\
\hline 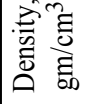 & $\begin{array}{l}\stackrel{\infty}{\infty} \\
\stackrel{i}{i}\end{array}$ & $\mid \begin{array}{l}\mathscr{O} \\
i\end{array}$ & $\stackrel{\infty}{i}$ & $\underset{\text { ¿ }}{\text { in }}$ \\
\hline 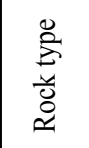 & 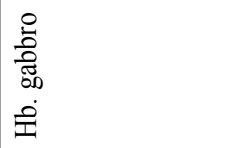 & 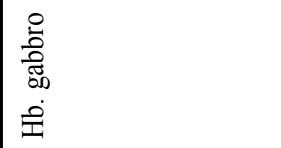 & 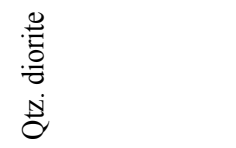 & 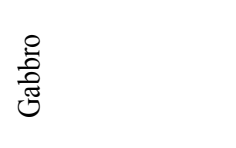 \\
\hline 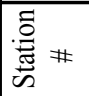 & $a$ & $\circ$ & $=$ & $\simeq$ \\
\hline
\end{tabular}


Engineering Geological Evaluation...

\begin{tabular}{|c|c|c|c|c|}
\hline 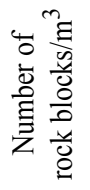 & $\begin{array}{ll}\vec{\infty} & \stackrel{+}{+} \\
\stackrel{9}{a} & \end{array}$ & 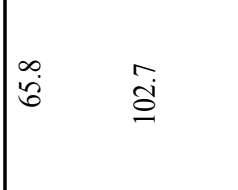 & $\begin{array}{ll}\vec{\infty} & \stackrel{\Xi}{\sigma}\end{array}$ & તี่ \\
\hline $\begin{array}{l}\dot{\overrightarrow{0}} \\
\dot{\vec{D}} \\
\dot{n}\end{array}$ & 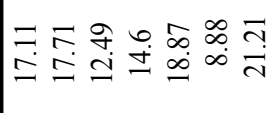 & 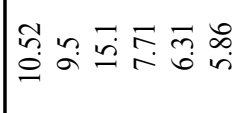 & 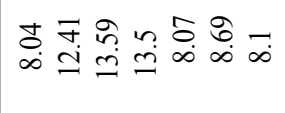 & 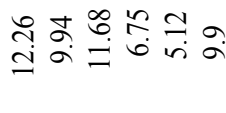 \\
\hline 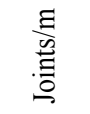 & 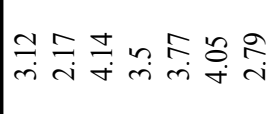 & 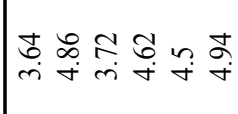 & ڤ & 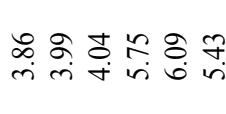 \\
\hline$\stackrel{\infty}{2} \approx$ & $\begin{array}{ll}- & 0 \\
2 & \stackrel{0}{\infty}\end{array}$ & 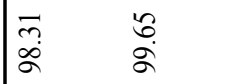 & $\begin{array}{ll}\infty & n \\
0 & \infty\end{array}$ & $\begin{array}{l}\stackrel{7}{*} \\
\stackrel{f}{a}\end{array}$ \\
\hline 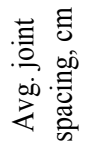 & 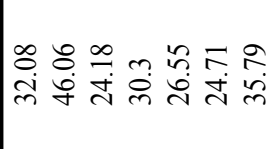 & 숭 & 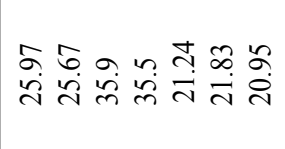 & 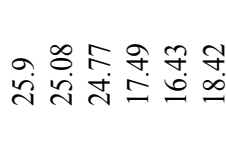 \\
\hline$\underset{\approx}{\approx}$ & 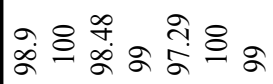 & \& & ৪ \& \& & 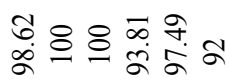 \\
\hline 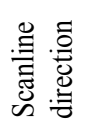 & 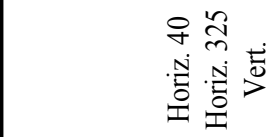 & 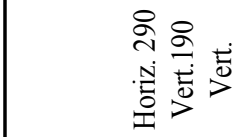 & 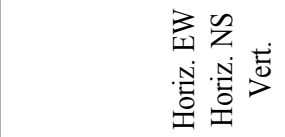 & 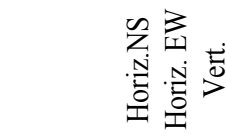 \\
\hline 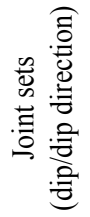 & 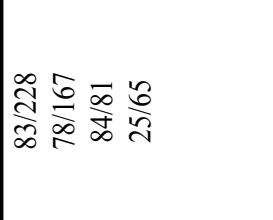 & 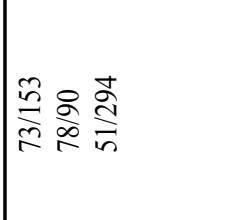 & $\stackrel{\infty}{\infty} \frac{\mathfrak{n}}{\infty} \frac{\bar{n}}{\curvearrowleft}$ & 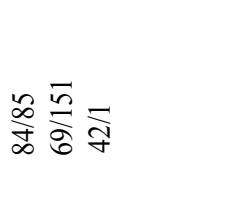 \\
\hline $0^{0} \stackrel{\pi}{\Sigma}$ & 芩 & $\overline{\vec{\sim}}$ & $\bar{p}$ & $\underset{\infty}{\infty}$ \\
\hline $3^{\infty}$ & 声 & 章 & 胃 & 界 \\
\hline 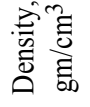 & $\grave{\sim}$ & d̦ & $\hat{\sigma}$ & $\overrightarrow{\mathrm{i}}$ \\
\hline 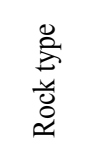 & 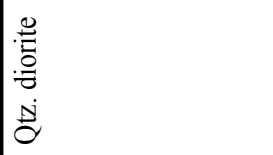 & 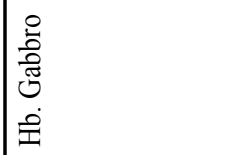 & $\begin{array}{l}0 \\
\stackrel{0}{0} \\
\stackrel{0}{0} \\
\dot{0} \\
\dot{1}\end{array}$ & $\begin{array}{l}\text { 윰 } \\
\text { हैं } \\
\dot{0} \\
\dot{1}\end{array}$ \\
\hline 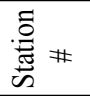 & $\because$ & \pm & $\because$ & 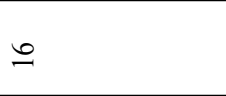 \\
\hline
\end{tabular}




\begin{tabular}{|c|c|c|}
\hline 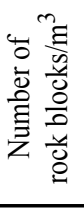 & $\begin{array}{ll}\grave{i} & \infty \\
\stackrel{0}{0}\end{array}$ & $\stackrel{m}{\stackrel{\infty}{n}} \quad \stackrel{\infty}{\stackrel{d}{n}}$ \\
\hline $\begin{array}{l}\vec{d} \\
\dot{\vec{d}} \\
\dot{\vec{n}}\end{array}$ & 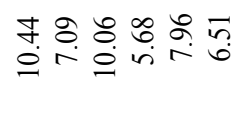 & 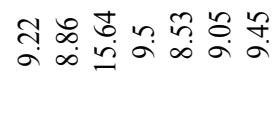 \\
\hline$\frac{\Xi}{\mathfrak{n}:}$ & 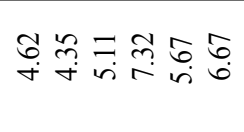 & 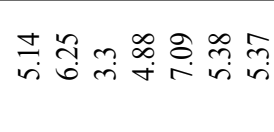 \\
\hline$\stackrel{\infty}{\gtrless} \underset{\sim}{\approx}$ & $\begin{array}{ll}\bar{\infty} & \infty \\
\stackrel{\infty}{\sigma} & \stackrel{8}{\circ}\end{array}$ & 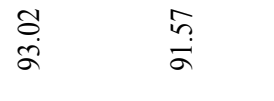 \\
\hline 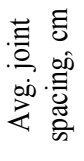 & 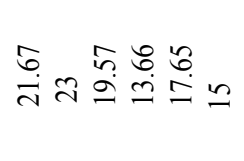 & 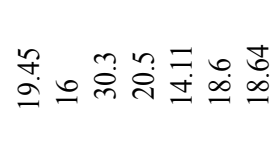 \\
\hline$\stackrel{2}{\approx}$ & 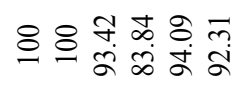 & 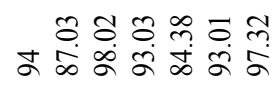 \\
\hline 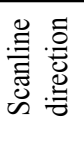 & 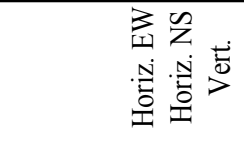 & 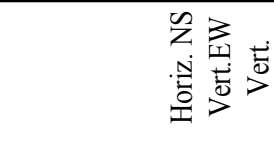 \\
\hline 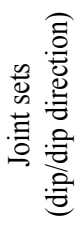 & 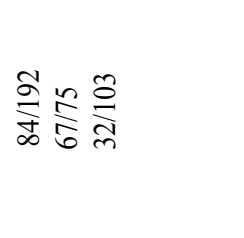 & 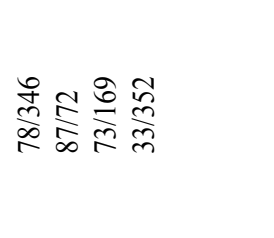 \\
\hline $0^{0} \stackrel{\tilde{\Sigma}}{\Sigma}$ & $\stackrel{\stackrel{+}{a}}{a}$ & 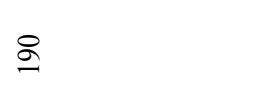 \\
\hline $3^{\infty}$ & $\stackrel{\Xi}{\Xi}$ & $=$ \\
\hline 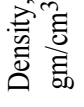 & 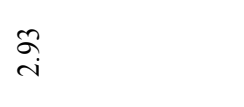 & $\stackrel{\mathrm{i}}{ }$ \\
\hline 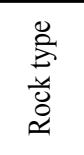 & 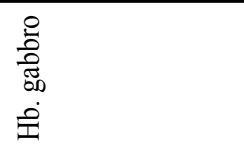 & 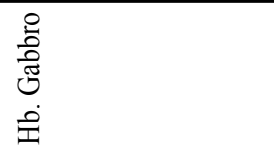 \\
\hline . & $=$ & $\stackrel{\infty}{.}$ \\
\hline
\end{tabular}




$$
\sigma_{\mathrm{c}}=(14+0.175 D) I_{S}
$$

\section{Weathering characteristics}

Weathering state of the rock masses is different from place to another. It ranges from stained fresh rock surfaces to more than half of the rock masses. Those locations characterized by high state of weathering in the site, form weakness regions in the area whereas the contaminated water, leachate and rainfall could percolate through it (Table 1).

\section{Discontinuities}

As the engineering properties of the prevailing joints system controls the propagation of the leachate and the flow and movement of the groundwater in the rock masses, a special attention was given to the study of the characteristics of the joints systems. These discontinuities are primarily responsible for the permeability of the available rock masses that decrease with increasing depth. A joints analysis was carried out at each station on the available rock masses in the site.

According to the important role of the joint intensities, a special attention was given to it in this study. At each station, the joints spacing was measured dually 1) across the dip direction of each major joint set, in order to reveal the effect of the local schistosity (Table 1), and 2) along a scanline of defined trend intersecting all joint planes crossing it. The following equation given by Palmstrom (1995) been used for the calculation of the number of blocks $/ \mathrm{m}^{3}, J_{v}$

$$
J_{v}=\frac{N_{x}}{L_{x}} \times \frac{N_{y}}{L_{y}} \times \frac{N_{z}}{L_{z}}
$$

where $N_{x}, N_{y}$, and $N_{z}$ are the number of discontinuities counted along the scanlines $\left(L_{x}, L_{y}\right.$, and $\left.L_{z}\right)$ perpendicular to each other. Application of GSI classification in the study area rock slopes is given in (Table 1).

Variation in the values of number of blocks $/ \mathrm{m}^{3}$ measured by methods 1 and 2 is related to the presence of random joints in the rock masses and the length and persistence of the joint planes.

The value of Rock Quality Designation (RQD) introduced by Deere et al. (1966) which indicates the degree of jointing in the rock mass was also calculated using the previous scanline results. The study shows that the density of the discontinuities varies with location within the rock mass. In general, jointing in the site is medium- to widely-spaced (Table 1). In some places in the site, the jointing is intense due to local schistosity. The attitude of such joint set(s) could 
form a possible path to the seepage that may result from the decomposition of the waste materials at the site, if not properly sealed from reaching the bedrocks and consequently contaminating the groundwater underneath in the vicinity of the disposal site.

Joint survey results given in (Table 1) show that: 1) the degree of fracturing in the available rock masses are excellent, and 2) the standard deviation of the joints/meter ratio is not close to zero, but indicate a significant spread or deviation about the mean (Davis, 1986). The standard deviation values vary with the joint/meter ratio, as shown in (Table 1). This means that it is possible to find blocky rock blocks of different sizes at each location. The probability approach allows the influence of the variation in values of all design parameters to be accurately accounted for in design; this is appropriate because in reality, all the parameters have a range of values. In contrast, deterministic analysis can only use sensitivity analysis of one parameter at a time to examine the influence of parameter values on the factor of safety.

\section{Rock masses geomechanics properties}

Bieniawski (1976) published Rock Mass Rating classification (RMR) system to classify the rock masses. Modifications had been done by Bieniawski (1989) to the classification to eliminate the limitations in the classification scheme for very poor quality rock masses and for unrealistic rating adjustment. The modified RMR classification system was used in the present study to classify the rock masses into different structural regions, which usually coincides with the major structural features (Hoek, 2000). The results of application of the Bieniawski classification (1989) are shown in (Table 2).

Hoek and Brown (1997) proposed a Geological Strength Index classification (GSI) based on the visual impression on the rock mass structure, on the basis of interlocking and surface condition of discontinuity. The GSI is based on personal judgment and field observations, however, it is more practical and easier to estimate the strength of the jointed rock masses from field observations. Hoek et al. (1998) modified the GSI by introducing a new category for highly heterogeneous rock masses and foliated/laminated rock mass structure.

\section{Water Balance and Leachate Generation}

One of the major problems associated with waste disposal is the generation of leachate. Leachate defined by Bell et al., (1993) as contaminated water which is produced when rainwater or groundwater flow through a landfill dissolving the soluble fraction of the waste. This occurs once the absorbent characteristics of the refuse are exceeded. The composition of leachate depends on the materials 


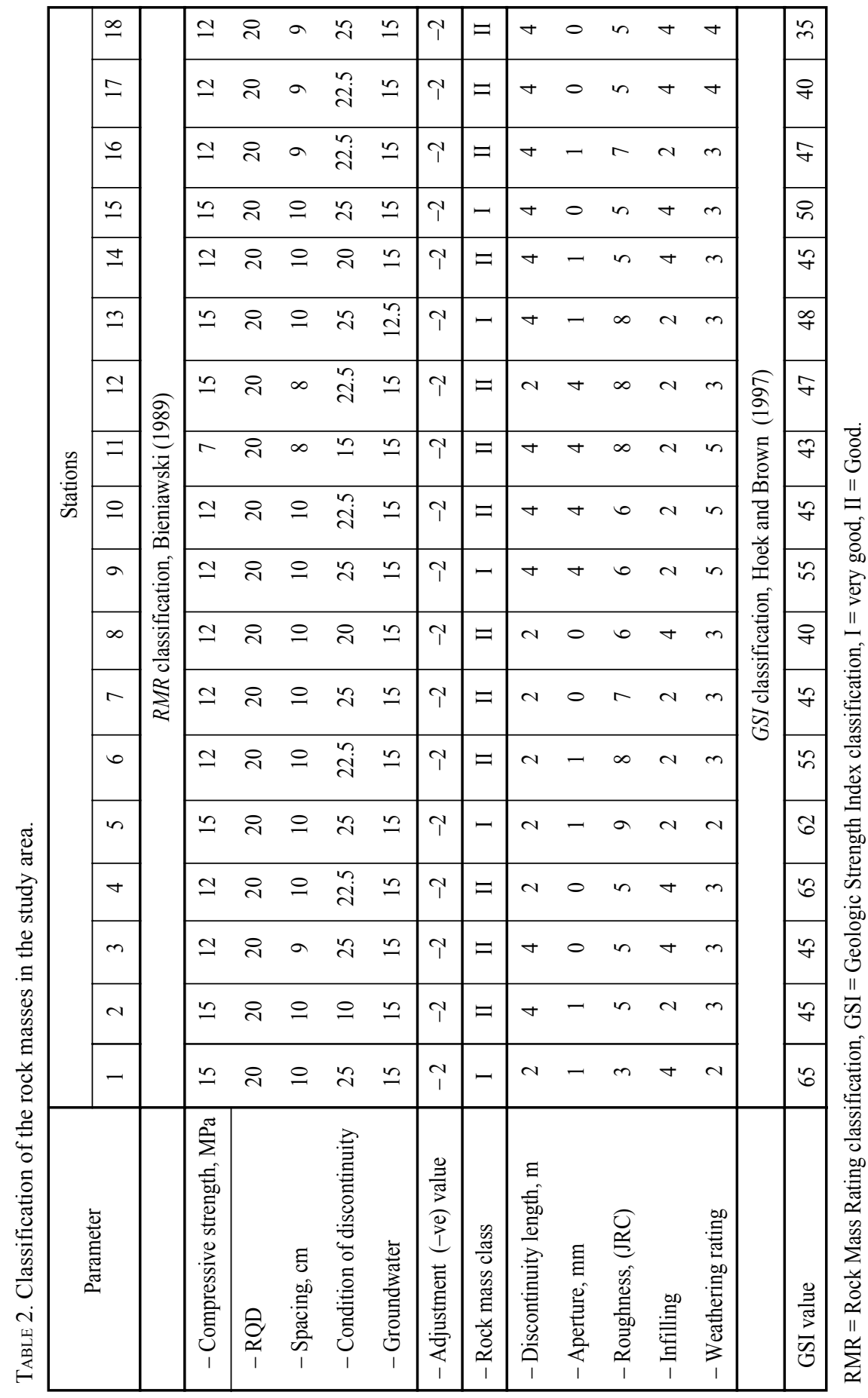


present in a landfill and the environmental conditions existing at the site. At many landfill sites, leachate has moved into the soil, groundwater, or surface water and this can cause considerable pollution. The most serious consequences will result when the control measures are inadequate. The leachate should be either contained within the landfill or removed for treatment (Tchobanoglous, et al., 1977). Ideally, leachate production should be kept to a minimum and monitored.

Generally, the largest contributor to leachate generation is the precipitation, which percolates through the waste, reacting with it during the process. In codisposal operations (where both liquids and solids are disposed of together), leachate generation is enhanced by the addition of liquid wastes.

\section{Water Balance}

An estimate of the volume of leachate that will be produced can be obtained from a water balance determination for a landfill, as estimated by Campbell (1983), which consists of an evaluation of liquid input and output from the site. The first part of a water balance calculation involves an assessment of the quantity of water entering a site, either as rainfall or by co disposal. The second part involves an evaluation of liquid retained in a landfill, as well as liquid lost due to evaporation and leachate flow, Water balance calculations, however, are complex.

A simplified water balance equation was used to derive the water balance for the landfill (Bell et al., 1993) as follows:

$$
L_{g}=p \times A+L_{c}-L_{S}
$$

where $L_{\mathrm{g}}$ is the amount of leachate generated, $P$ is the percentage percolation from precipitation, $A$ is the area of the landfill, $L_{\mathrm{c}}$ is the quantity of liquid co disposal and $L_{\mathrm{S}}$ is the quantity of liquid retained in storage which is primarily the absorptive capacity of the waste.

One of the problems involved concerns the determination of the absorptive capacity of the waste material. Absorptive capacity is influenced by the composition of the waste, and the method of waste treatment prior to placing it in the disposal site.

\section{Discussion}

A common philosophy is to apply the multiple barrier concept, which means that more than one medium or mechanism, physical or chemical, prevent or retard release and migration of the hazardous waste. 
The basic principle of proper location and design of terrestrial repositories is to isolate the waste from the biosphere such that the concentration of toxic substances in the groundwater, in this case, does not exceed the maximum values stipulated by national authorities. From the engineering point of view, two major principles can be considered for location of repositories, i.e. a) "dry" conditions with repository above the groundwater surface and designed so that it is not infiltrated and percolated by water, and b) "wet" conditions with the repository located so that the amount of percolating groundwater is at minimum and becomes discharged into large recipients, where the concentrations will be small.

The first principle, which implies that the dissolution of the waste and contamination of the groundwater are negligible, requires very shallow location of the repository in most areas, except for desert, but problems can be identified: Climatic alterations must be foreseen, such as change from arid to humid conditions. Location in rock above the present groundwater level may represent an additional risk if the repository is planned to host hazardous wastes. The thermal pulse will also induce rock strain that may cause permanent increase in fracture apertures and hence an increased hydraulic conductivity.

The second principle is expected to be of general use and there are numerous examples accordingly planned but not yet constructed repositories.

Both principles imply that the stability of the rock is of fundamental importance for the function of repositories, rock mechanics and stress conditions in the earth crust are considered in localizing and designing rock repositories, for more details refer to Pusch (1994).

More effective isolation of the waste is obtained by surrounding it with a low-permeable substance like concrete, and/or clay which also offer a suitable chemical environment and, especially for clay, its rheological properties like ductility and ability to undergo stress relaxation for maintaining homogeneity and uniform embedment. Clay and/or geosynthetic textiles are also used as an impermeable layer between the compacted solid waste layers. Geosynthetics (geotextiles and geomembranes) are used in between wastes layers. Geosynthetic clay liners (or GCLs) are the newest subset within geosynthetic materials. They are rolls of factory fabricated thin layers of bentonite clay sandwiched between two geotextiles or bonded to a geomembrane. Structural integrity is maintained by needle punching, stitching or physical bonding. They are used as a composite component beneath a geomembrane or by themselves as primary or secondary liners.

The most effective isolation of waste is obtained by encapsulating it in longlived containers, "canisters", which are embedded in concrete or clay and em- 
placed or clay and emplaced in low-permeable rock and excavated so as to minimize mechanical disturbance.

Testing of groundwater has used mostly constant-rate withdrawal methods for higher conductivity rocks and pulse tests for lower conductivities. These tests use transient flow methods, which include storage terms, storage reflecting the compressibility and deformability of the water and rocks. There are four major methods of testing crystalline rock (constant-rate, constant-pressure, pressure-recovery, and pulse/slug). Doe (1987) has discussed these tests and permeability of the crystalline rocks in details.

There are two approaches to design the sampling programs. First discretezone testing (DZ), which is used when a specific zone, such as a fracture zone or aquifer, is the main concern, second fixed-interval-length testing (FIL) which is used when the testing is meant to provide a statistical sample of the rock's hydraulic properties. This FIL test reflects the frequency of fractures and the distribution of conductivity (Doe, 1987). The sampling strategies for crystalline rocks looking at the inference of fracture spacing and transmissivity distributions from a series of well tests, and the problem of channelized flow.

To estimate the amount of land area required for preliminary landfill site planning, Tchobanoglous et al. (1977) introduced a chart concerning the relation of the compacted density of solid wastes and the average depth of compacted solid waste. The following equations are used to calculate the required area and volume/year:

$$
\text { Generation rate }=\text { number of population } \times \text { solid waste generation }
$$

Site volume required / day $=\frac{\text { Generation rate }}{\text { Compacted density of solid wastes }}$

$$
\text { Area required year }=\frac{\text { Volume required } / \text { day } \times 365 \text { days }}{\text { Average depth of compacted solid waste }}
$$

$$
\text { Lifetime of the landfill }=\frac{\text { Available site volume }}{\text { Site volume required } / \text { year }}
$$

Tchobanoglous et al. (1977) introduced a method to calculate the expected generated leachate from the amount of dumped solid wastes. Such set of calculations is required to be commenced for Malkan-1 site before using it.

The location of the site, easy access, utility access, good quality condition of the hosting rock foundations, limited access to the surrounding wadis, large size are factors that give privileges to the site. 


\section{Conclusion and Recommendations}

1. The rock masses in the studied area vary in their engineering characteristics from location to another. However, the quality of the rock masses is ranging between very good to good quality according to the latest classification systems. Accordingly, the site is suitable to be used as a landfill. Nevertheless, weathering state of the rocks could be the possible zones of weakness in the rock masses, which could result in transmitting a leachate or contaminated groundwater through it to the main Wadi. Accordingly, these weathered areas should be first excavated, and then shotcrete allover the rock surface at the site entrance.

2. The lack of major faults in the proposed site indicates no possible leakage through major faults planes (if any). In addition, the located small-scale faults and shear zones has no significant role in transmitting any leachate or contaminated water out of the site outlet to the main wadi, unless an unobserved major fault is detected.

3. Because of the major trend of the joint systems and dikes (northeast southwest), and the general slope of the site toward east, this may lead to a minor seepage problem. In order to prevent that from taking place and for safety reasons, the proposed waste disposal site should be monitored continuously along its eastern side, north and south sides of the entrance.

4. Rainfall information should be provided, in addition to the estimated quantity of liquid waste to calculate the possible quantity of leachate generation through the wastes in the site.

5. In crystalline rocks (high grade metamorphic, and igneous rocks) characterized by low permeability, and the flow is primarily in a system of fractures. The design of well testing programs for low permeability, fractured rock has two elements A. the selection of the well tests methods, and B. the design of the sampling program. Rocks where flow is primarily in fractures may be extremely heterogeneous in their hydrologic properties. Thus the test methods chosen must be capable of providing data over a broad range of hydraulic conductivities. The heterogeneity also means that the sampling program must be designed to directly test or infer the properties of the conduits in the rock mass whether or not they are intersected by the borehole.

6. Site geology and hydrology should be thoroughly characterized by qualified professionals to properly locate, design, construct, operate and monitor solid and hazardous waste facilities. The quantities and nature of chemicals used in manufacturing and households must be addressed, and the waste streams reduced and changed, to reduce the risk of future soil and groundwater contamination. Substitution of less dangerous materials in manufacturing, re- 
cycling and the segregation of incompatible wastes, and the development of alternative treatment and disposal options are necessary. Liners, tanks, and monitoring systems should use technologies to minimize the potential for problems and to provide for early leak detection.

7. Site geologic conditions and potential uses of contaminated aquifers must be considered to design cost-effective remedial measures.

8. Those locations at stations characterized by a large number of rock blocks/ $\mathrm{m}^{3}$ need a thorough sealing underneath the lining of the landfill site.

9. Further detailed investigations are recommended, in order to trace the possible fluid flow path through the rock masses joint systems. Detailed in-situ tests and investigations about i) the effective permeability and ii) the in-situ hydraulic conductivity of the jointed rock masses, in addition to iii) rock slope stability of the new rock slope cuts of the site sides.

\section{References}

ASTM (1995) Standard test method for specific gravity and absorption of coarse aggregate. C127-88 (reapproved 1993). Annual book of ASTM standards, 04.02: 64-68.

Bell, F.G., Sillito, A.J. and Jermy, C.A. (1993) Landfills and associated leachate in the greater Durban area: two case histories. The engineering geology of waste storage and disposal. Conference of the Engineering Group of the Geological Society of London, 6-9 September, $1-17$.

Bieniawski, Z.T. (1975) The point load test in geotechnical practice. Engineering Geology, 9: 111.

Bieniawski, Z.T. (1976) Rock mass classification in rock engineering. In: Exploration for rock engineering, proc. of the symp., (ed. Z. T. Bieniawski), Cape Town: Balkema. 1: 97-106.

Bieniawski, Z.T. (1989) Engineering rock mass classification. New York: Wiley, p. 251.

Broch, E. and Franklin, J.A. (1972) The point load strength test. Int. J. Rock Mech. and Mining Sci., 9: 669-697.

Campbell, D. (1983) Understanding water balance in landfill sites. Waste Management, 11: 594601.

Davis, J.C. (1986) Statistical and data analysis in geology. $2^{\text {nd }}$ edition. John Wiley \& Sons, p. 646.

Deere, D.U., Hendron, Jr., A.J., Patton, F.D. and Cording, E.J. (1966) Design of surface and near-surface construction in rock. Failure and breakage of rock, $8^{\text {th }}$ US Symposium on Rock Mechanics, Minnesota, AIME, 237-302.

Doe, T.W. (1987) Fluid flow and waste isolation in rock masses. $6 \mathrm{t}^{\mathrm{h}}$ International Congress of Rock Mechanics, Montreal, Canada, 1377-1410.

Hoek, E. (2000) Practical rock engineering. Internet edition, p. 313.

Hoek, E. and Brown, E.T. (1997) Practical estimates of rock mass strength. Int. J. Rock Mech. Min. Sci., 34(8): 1165-1186.

Hoek, E., Marinos, P. and Benissi, M. (1998) Application of the geological strength index (GSI) classification for very weak and sheared rock masses: the case of the Athens schist formation. Bull. Eng. Geol. Environ., 57: 151-160. 
Langer, M. (1995) Engineering geology and waste disposal. IAEG Commission report No. 14. Bulletin of the International Association of Engineering Geologists, 51: 6-29.

Mokhtar, T.A., Basyoni, M.H. and Sadek, H.S. (1995) Siting of a new landfill for solid wastes in the Kingdom of Saudi Arabia. In Bell, R.S. (Complier): Proc. symp. on the application of geophysics to engineering and environmental problems. April 23-26, Orlando, FL., 835842.

Mokhtar, T.A., Sadek, H.S., Basyoni, M.H., Mofti, A., Alwash, M.A. and Sadagah, B.H., (1996) Determination of the buried wastes sites at Wadi Ibrahim area and study of its effect on the groundwater. Supported Proj. No. M. O. 30-14, King Abdulaziz University, Jeddah, Saudi Arabia, p. 350.

Moore, T.A. and Al-Rehaili, M.H., (compilers) (1989) Geologic map of he Makkah quadrangle, sheet 21D, Kingdom of Saudi Arabia. Geoscience map GM-107c. Directorate General of Mineral Resources, Jeddah, Saudi Arabia.

Morfeldt, C.O. and Langer, M. (1989) Problems of underground disposal of waste. IAEG Commission report No. 14. Bulletin of the International Association of Engineering Geologists, 39: 3-24.

Palmstrom, A. (1995) Rmi-a rock mass characterization system for rock engineering purposes. Ph.D. thesis, Oslo University, Norway, p. 382.

Pusch, R. (1994) Waste disposal in rock. Developments in Geotechnical Engineering, 76. Elsevier Publishers, p. 490.

Tchobanoglous, G., Theisen, H. and Eliassen, R. (1977) Solid wastes, engineering principles and management issues. McGraw Hill, p. 621. 


\title{
دراسة تقييم جيولو جية هندسية لموقع نفايات صلبة مقترح
}

\author{
بهاء الدين هاشم صدقه \\ قسم الجيولوجيا الهندسية والبيئية، كلية علوم الأرض، جاءئ هامعة الملك عبد العزيز

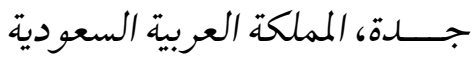

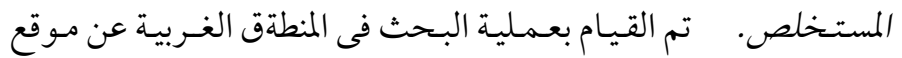

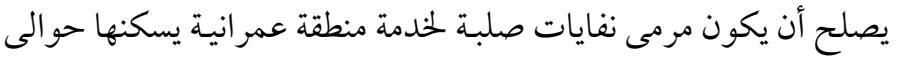

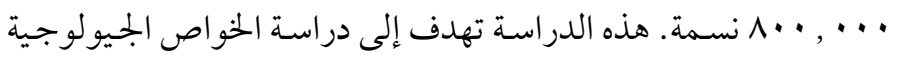

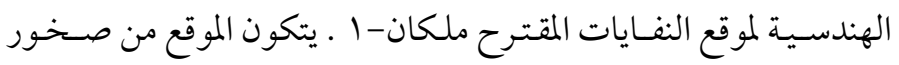

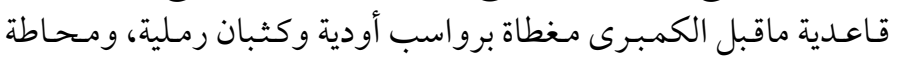

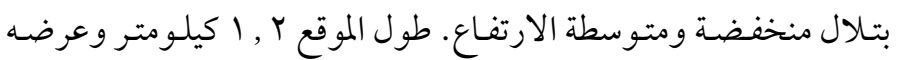

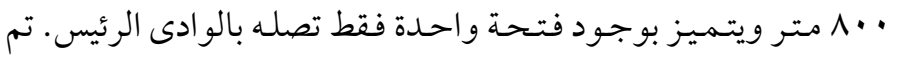

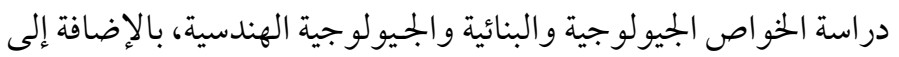

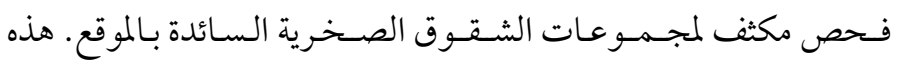

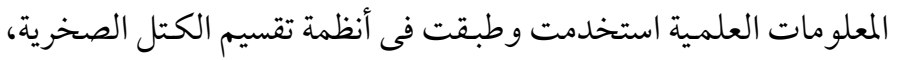
وإستنتج منها صلاحية الموقع للاستخدام كمرمى للنفايات الصلبة. 